\title{
THE EUROPEAN LARGE AREA ISO SURVEY: ELAIS
}

\author{
S.J. OLIVER \\ Imperial College of Science Technology and Medicine \\ Astrophysics Group \\ Blackett Laboratory \\ Prince Consort Rd. \\ London \\ $S W 21 B Z$ \\ s.oliver@ic.ac.uk
}

\begin{abstract}
. ${ }^{1}$
I describe a European collaborative project to survey $\sim 20$ square degrees of the sky at $15 \mu \mathrm{m}$ and $90 \mu \mathrm{m}$ with ISO. This is the largest open time project being undertaken by ISO. The depth and areal coverage were designed to complement the various Guaranteed Time surveys. The main science thrust is to explore star formation in galaxies to a much higher redshift than was probed by IRAS. We expect to detect around 8000 extragalactic objects and a similar number of Galactic sources. The maps and source catalogues will represent a major legacy from ISO, inspiring follow up work for many years to come.
\end{abstract}

\section{Introduction}

The Infrared Space Observatory (ISO) will be the only major infrared mission for the next decade. Although the satellite was principally designed as an observatory the case for devoting a substantial amount of the mission time to surveys was overwhelming.

The Infrared Astronomical Satellite (IRAS) had enormous success arising principally from its survey products (particularly the Point Source

\footnotetext{
${ }^{1}$ To appear in "Cold Gas at High Redshift" Eds: Malcolm Bremer, Huub Rottgering, Paul van der Werf and Chris Carilli. Pubs: Kluwer
} 
Catalog and the Faint Source Catalog). Perhaps most significant was the discovery of a whole new class of objects with enormously high far infrared luminosity [notably F10214+4724 (Rowan-Robinson et al., 1991) and P09104+4109 (Kleinmann et al., 1988)]. As well as discovering new objects, IRAS demonstrated the benefit of selecting objects in the far infrared. This wave-band is not sensitive to dust obscuration which biases optically selected samples. The emission arises from thermally heated dust and thus complements studies of emission directly from star-light, gas, or AGN engines.

The sensitivity of ISO is orders of magnitude better than IRAS. Using it as a survey instrument will thus allow us to explore IRAS-like populations to higher redshift and possibly unveil new classes of objects or unexpected phenomena.

This paper outlines the open time survey which is a collaborative venture between fifteen European Institutes; the PI being M. Rowan-Robinson, Co-Is being: C. Cesarsky, L. Danese, A. Franceschini, R. Genzel, A. Lawrence, D. Lemke, R. McMahon, G. Miley, S. Oliver, J-L. Puget and B. Rocca-Volmerange. Many other people are also heavily involved.

\section{Science Goals}

While it is impossible to predict all the scientific benefits of such a large project, I outline some of the key issues that we hope to address. A major theme is the detection of high redshift galaxies.

\subsection{EPOCH OF GALAXY FORMATION}

The search for galaxies at high redshift to uncover the formation epoch is one of the holy grails of cosmology. The failure to detect high redshift objects in optical surveys, particularly using Ly $\alpha$, has two competing explanations. The first is that early galaxies contain a large dust component which obscures the optical emission. E.g. if elliptical galaxies underwent a massive burst of star-formation between $2<z<5$, they would be observable in the far infrared since massive stars produce both dust and the UV to heat it, and may look like F10214 (Elbaz et al., 1992). Alternatively galaxies may have been formed by the assembly of constituents which are individually too faint to detect. This survey will provide a powerful discrimination between these two hypotheses, since we would detect optically obscured galaxies but not low luminosity proto-galaxies. 


\subsection{STAR FORMATION IN SPIRAL GALAXIES AT HIGH REDSHIFT}

The main extra-galactic population detected by IRAS was galaxies with high rates of star formation. Their far infrared emission arises from dust heated by young stellar populations. These objects are now known to evolve with a strength comparable to AGN (Oliver el al., 1995). The distance to which these objects were visible by IRAS was, however, insufficient to determine the nature of their evolution. The sensitivity of ISO will allow us to detect these objects at much higher redshifts and thus obtain greater understanding of the cosmological evolution of star formation.

\subsection{ULTRA AND HYPER-LUMINOUS I-R GALAXIES AT HIGH $Z$}

IRAS uncovered a population with enormous far infrared luminosities, $L_{\mathrm{FIR}}$ $>10^{12} L \odot$. This far infrared emission represents the bulk of the bolometric luminosity of these objects which is comparable to that of AGN. The local space density of these objects, however, exceeds that of optically selected AGN, implying this population is a more energetically significant component of the Universe. For these reasons this population has been carefully studied. The energy source in these objects is still disputed. While most of these objects appear to have an AGN it is argued that star formation could provide most of the energy. Interestingly, most of these objects appear to be in interacting or merging systems, suggesting a triggering mechanism. Exploration of these objects at higher redshift will have particular significance for models of AGN/galaxy evolution.

\subsection{EMISSION FROM DUSTY TORI AROUND AGN}

Unified models of AGN suggest that the central engine is surrounded by a dusty torus. Optical properties are then dependent on the inclination angle of this torus. The far infrared emission from the torus will be less sensitive to the viewing angle. Thus a far infrared selected sample of AGN will be more uniform than an optically selected sample and the far infrared properties of these will place important constraints on unification schemes. AGN are known to be strongly evolving and this sample will tell us about the evolution of the tori. Also, we will be able to detect dust emission from tori in 'face-on' AGN which would not be detected in the optical.

\subsection{DUST IN NORMAL GALAXIES TO COSMOLOGICAL DISTANCES}

Faint optical redshift surveys find surprisingly few galaxies beyond $z=0.5$. One possible explanation for this is a dust fraction that increases with $z$. Emission from the cool interstellar 'cirrus' dust in normal galaxies will be 
detectable in our survey to much greater distances than were accessible with IRAS, so we will be able to examine the dust content to higher $z$.

\subsection{CIRCUMSTELLAR DUST EMISSION FROM GALACTIC HALO STARS}

The deep stellar number counts provided by this survey will be relatively unaffected by Galactic extinction and may provide, amongst other things, improved estimates of the halo/disk population ratios.

\subsection{NEW CLASSES OF GALACTIC AND EXTRA-GALACTIC OBJECTS}

F10214 was at the limit of IRAS sensitivity and new classes of objects may well be discovered at the limit of the ISO sensitivity. The lensing phenomenon which made F10214 detectable by IRAS may become more prevalent at fainter fluxes, increasing the proportion of interesting objects. Current predictions suggest we would not expect to detect Galactic Brown Dwarfs but unexpected Galactic objects may be discovered.

\subsection{CLUSTERING PROPERTIES}

The volume of this survey is comparable to that surveyed by the entire IRAS Point Source Catalog. The median redshift will be much higher. We will thus be in a position to examine the evolution of clustering strength, giving perhaps the most direct test of the gravitational instability picture of structure formation.

\section{Survey Definition}

As with any time-constrained survey we had to balance factors such as depth, wavelength and areal coverage. To complement Guaranteed Time deep ISO CAM surveys (Franceschini et al., 1995) we decided to sacrifice depth at the shorter wavelength for increased areal coverage. This section describes the rationale behind the choices we made for: wavelengths, depths and areas.

\subsection{WAVELENGTH AND SENSITIVITIES}

We initially proposed to survey at three wavelengths to give useful colour information over a long wavelength baseline but were required by the OTAC to restrict ourselves to two. At the longer ISO wavelengths we pick up star forming galaxies. Consideration of the SED of these galaxies together with the capabilities of the ISO PHOT instrument suggested that the optimal sensitivity to these objects would be obtained using the C100 detector with $90 \mu \mathrm{m}$ filter. At shorter wavelengths ISO is more sensitive to AGN emission. 
TABLE 1. Survey parameters for a single raster

$\begin{array}{lcc}\text { Instrument } & \text { CAM } & \text { PHOT } \\ \text { Filter } & \text { LW-3 } & 90 \\ \lambda_{0} / \mu \mathrm{m} & 15 \pm 3 & 95.1 \pm 26 \\ \text { Detector } & \text { LW-l Si }(+\mathrm{Ga}) & \mathrm{C} 100 \mathrm{Ge}: \mathrm{Ga} \\ \text { AOT } & \mathrm{CAM} 01 & \text { PHOT } 22 \\ \text { Pixel Size } & 5.6^{\prime \prime} & 43.5^{\prime \prime} \\ \text { Pixels } / \text { frame } & 32 \times 32 & 3 \times 3 \\ \text { Frame size } & 180^{\prime \prime} & 135^{\prime \prime} \\ \delta x, \delta y & 90^{\prime \prime}, 180^{\prime \prime} & 130^{\prime \prime}, 130^{\prime \prime} \\ \text { Raster Points } & 28 \times 14 & 20 \times 20 \\ \text { Raster size } & \left(42^{\prime}\right)^{2} & \left(43.3^{\prime}\right)^{2} \\ 5 \sigma \text { Sensitivity } & 1.7 \mathrm{mJy} & 15 \mathrm{mJy}\end{array}$

Consideration of the ISO CAM sensitivities, AGN SEDs and avoidance of frequencies in atmospheric windows lead us to select the CAM LW-3 filter centred at $15 \mu \mathrm{m}$.

The limited resolution but high sensitivity of ISO at long wavelengths means that the Galactic Cirrus confusion limit is reached with very short integration times. This confusion limit thus defined our PHOT integration. We decided to use a similar total observation time for both instruments. Table 1 summarises the two observing modes used.

\subsection{AREAS}

The allocated observing time allowed 37 rasters as described above. The choice of where to distribute these on the sky was governed by a number of factors. Firstly we decided not to group these all in a single contiguous region of the sky. Had we done so we may have had difficulty distinguishing evolutionary effects from local large scale structures. Distributing the survey areas across the sky also has advantages for follow up work. Cirrus confusion is a particular problem, so we selected regions with low IRAS $100 \mu \mathrm{m}$ intensities $\left(I_{100}<1.5 \mathrm{MJy} / \mathrm{sr}\right)$, using the maps of (Rowan-Robinson et al., 1991b). To avoid conflict with other ISO observations we further restricted ourselves to regions of high visibility over the mission lifetime $(>25 \%)$. To avoid unnecessarily high Zodiacal backgrounds we only selected regions with high Ecliptic latitudes $\left(|\beta|>40^{\circ}\right)$. Finally it was essential to avoid saturation of the CAM detectors so we had to avoid any bright IRAS $12 \mu \mathrm{m}$ sources. These requirements led us to selecting the four areas 
TABLE 2. Summary of Areas. The first four areas comprise the main survey made up from $43^{\prime} \times 43^{\prime}$ rasters. One raster in N3 will be repeated. The final 6 areas are single smaller rasters $24^{\prime} \times 24^{\prime}$

\begin{tabular}{|c|c|c|c|c|c|c|}
\hline Area & Rasters & \multicolumn{2}{|c|}{$\begin{array}{c}\text { Nominal Coordinates } \\
\text { J2000 }\end{array}$} & $\begin{array}{c}\left\langle I_{100}\right\rangle \\
/ \mathrm{MJysr}^{-1}\end{array}$ & $\begin{array}{c}\text { Visibility } \\
/ \%\end{array}$ & $\beta$ \\
\hline N1 & $3 \times 3$ & $16^{h} 08^{m} 44^{s}$ & $+56^{\circ} 26^{\prime} 30^{\prime \prime}$ & 1.2 & 98.0 & 73 \\
\hline $\mathrm{N} 2$ & $4 \times 2-1$ & $16^{h} 39^{m} 34^{s}$ & $+41^{\circ} 15^{\prime} 34^{\prime \prime}$ & 1.1 & 58.7 & 62 \\
\hline N3 & $3 \times 3$ & $14^{h} 28^{m} 26^{s}$ & $+32^{\circ} 25^{\prime} 13^{\prime \prime}$ & 0.9 & 26.9 & 45 \\
\hline $\mathrm{S} 1$ & $4 \times 3$ & $00^{h} 38^{m} 24^{s}$ & $-43^{\circ} 32^{\prime} 02^{\prime \prime}$ & 1.1 & 32.4 & -43 \\
\hline Lock. 3 & 1 & $13^{h} 34^{m} 36^{s}$ & $+37^{\circ} 54^{\prime} 36^{\prime \prime}$ & 0.9 & 17.3 & 44 \\
\hline Sculptor & 1 & $00^{h} 22^{m} 48^{s}$ & $-30^{\circ} 06^{\prime} 30^{\prime \prime}$ & 1.3 & 27.5 & -30 \\
\hline TX1436 & 1 & $14^{h} 36^{m} 43^{s}$ & $+15^{\circ} 44^{\prime} 13^{\prime \prime}$ & 1.7 & 22.2 & 29 \\
\hline $4 \mathrm{C} 24.28$ & 1 & $13^{h} 48^{m} 15^{s}$ & $+24^{\circ} 15^{\prime} 50^{\prime \prime}$ & 1.4 & 16.8 & 33 \\
\hline VLA 8 & 1 & $17^{h} 14^{m} 14^{s}$ & $+50^{\circ} 15^{\prime} 24^{\prime \prime}$ & 2.0 & 99.8 & 73 \\
\hline Phoenix & 1 & $01^{h} 13^{m} 13^{s}$ & $-45^{\circ} 14^{\prime} 07^{\prime \prime}$ & 1.4 & 36 & \\
\hline
\end{tabular}

detailed in Table 2. A further 6 areas were selected as being of particular interest to warrant a single small $\left(24^{\prime} \times 24^{\prime}\right)$ raster. These were chosen either because of existing survey data or because the field contained a high redshift object and were thus more likely to contain high redshift ISO sources. These 6 regions are also described in Table 2 .

\section{Expectations}

IRAS luminosity functions and model SED of star-bursts, AGN and normal galaxies and F10214 like objects together with simple pure luminosity evolution models have been used to predict the number of extra-galactic object we expect to see (Pearson \& Rowan-Robinson, 1995). This simple model predicts 5000 star-bursts (20\% detected in both bands, $30 \% z>1$ ), 650 AGN (5\% detected in both bands, $23 \% z>1$ ), 2300 normal galaxies (30\% detected in both bands) and 4 F10214 like objects. Models including a dusty phase in elliptical galaxy formation would predict higher numbers. We would also expect of order 10000 stars.

\section{Science Products}

The products we will provide to the community are source catalogues together with catalogue associations and maps at both ISO wavelengths. We anticipate these will be available a year after the end of the ISO mission 
(i.e. May 1998). A WWW page will be on line in the near future ${ }^{2}$ to keep the community abreast of the progress of the survey, a link to this will be found on http://icstar5.ph.ic.ac.uk/

\section{References}

Elbaz, D. et al. (1992) Astr. Astrophys.,Vol. no. 265, pp. L29-L32

Franceschini A., Cesarsky,C Rowan-Robinson, M., (1995) In 'Near-IR Sky Survey' San Maniato (Pisa) Memorie della Societa Astronomica Italiana (in press)

Kleinmann, S.G. et al. (1988) Astophys. J. Vol. no. 328, pp. 161-169

Oliver, S., et al. (1995), In Wide-Field Spectroscopy and the Distant Universe, Maddox, S.J., Aragon-Salamanca, A. eds, Proceedings of the 35th Herstmonceux Conference, World Scientific. p. 264

Pearson, C., Rowan-Robinson, M. et al. (1995) Mon. Not. R. Astro. Soc., (in press)

Rowan-Robinson, M. et al. (1991) Nature, Vol. no. 351, pp. 719-721

Rowan-Robinson, M. et al. (1991) Mon. Not. R. Astro. Soc., Vol. no. 249, pp. 729-741

${ }^{2}$ available now http://artemis.ph.ic.ac.uk/ 